\title{
LAGRANGIAN SUBMANIFOLDS OF THE COMPLEX HYPERBOLIC QUADRIC
}

\author{
JOERI VAN DER VEKEN AND ANNE WIJFFELS
}

\begin{abstract}
We consider the complex hyperbolic quadric $Q^{* n}$ as a complex hypersurface of complex anti-de Sitter space. Shape operators of this submanifold give rise to a family of local almost product structures on $Q^{* n}$, which are then used to define local angle functions on any Lagrangian submanifold of $Q^{* n}$. We prove that a Lagrangian immersion into $Q^{* n}$ can be seen as the Gauss map of a spacelike hypersurface of (real) anti-de Sitter space and relate the angle functions to the principal curvatures of this hypersurface. We also give a formula relating the mean curvature of the Lagrangian immersion to these principal curvatures. The theorems are illustrated with several examples of spacelike hypersurfaces of anti-de Sitter space and their Gauss maps. Finally, we classify some families of minimal Lagrangian submanifolds of $Q^{* n}$ : those with parallel second fundamental form and those for which the induced sectional curvature is constant. In both cases, the Lagrangian submanifold is forced to be totally geodesic.
\end{abstract}

\section{INTRODUCTION}

The complex quadric $Q^{n}$ is a Kähler-Einstein manifold, which can be seen in several different ways, for example as a complex hypersurface of the complex projective space $\mathbb{C} P^{n+1}$, as the Grassmannian manifold of oriented 2-planes in $\mathbb{R}^{n+2}$ or as the homogeneous space

$$
Q^{n}=\frac{\mathrm{SO}(2+m)}{\mathrm{SO}(2) \times \mathrm{SO}(m)} .
$$

Minimal Lagrangian immersions into $Q^{n}$ were studied for example in [8], [9], [3] and [7], by identifying them with Gauss maps of isoparametric hypersurfaces of the unit sphere $S^{n+1}(1)$. The relation between the geometric invariants of (not necessarily minimal) Lagrangian submanifolds of $Q^{n}$ and (not neccesarily isoparametric) hypersurfaces of $S^{n+1}(1)$ was stated in full generality in [17].

In the present paper, we study Lagrangian submanifolds of the complex hyperbolic quadric. This is the homogeneous space

$$
Q^{* n}=\frac{\mathrm{SO}^{0}(2, m)}{\mathrm{SO}(2) \times \mathrm{SO}(m)}
$$

which can be identified with the Grassmannian manifold of negative definite oriented 2-planes in the indefinite vector space $\mathbb{R}_{2}^{n+2}$. It is known from [10] that we can see $Q^{* n}$ as a complex hypersurface of the complex anti-de Sitter space $\mathbb{C} H_{1}^{n+1}$. We will discuss this immersion in more detail in Section 2. In particular, we explain how the induced geometric structures make $Q^{* n}$ into a homogeneous (Riemannian) Kähler-Einstein manifold, carrying a family of local almost product structures. The complex hyperbolic quadric $Q^{* 1}$ of complex dimension 1 is isometric to the hyperbolic plane $H^{2}(-2)$ of constant Gaussian curvature -2, whereas the complex hyperbolic

2010 Mathematics Subject Classification. Primary: 53C42; Secondary: 53D12; 53B25.

The first author is supported by the Excellence Of Science project G0H4518N of the Belgian government and both authors are supported by project 3E160361 of the KU Leuven Research Fund. 
JOERI VAN DER VEKEN AND ANNE WIJFFELS

quadric $Q^{* 2}$ of complex dimension 2 is isometric to the Riemannian product $H^{2}(-4) \times H^{2}(-4)$. The latter isometry follows for example from [4], since $Q^{* 2}$ is a real 4-dimensional Einstein manifold whose minimal sectional curvature is -4 , while it does not have constant sectional curvature, nor constant holomorphic sectional curvature.

Submanifolds of the complex hyperbolic quadric, in particular curves and real hypersurfaces, have recently attracted attention of several geometers, see for example [16], [5] and [6]. In the present paper, we deal with Lagrangian submanifolds of $Q^{* n}$. Inspired by the situation in $Q^{n}$, where Lagrangian immersions correspond to Gauss maps of spherical hypersurfaces, we define the Gauss map of a spacelike hypersurface of the anti-de Sitter space $H_{1}^{n+1}(-1)$ as a map into $Q^{* n}$, which turns out to be a Lagrangian immersion. Conversely, Theorem 4.4 states that one can see any Lagrangian submanifold of $Q^{* n}$, at least locally, as the Gaussian image of a hypersurface of $H_{1}^{n+1}(-1)$. This hypersurface will not be unique, as parallel hypersurfaces have the same Gauss maps. Nevertheless, Theorem 4.4 also gives a relation between combinations of the principal curvatures of a hypersurface, which do not change when replacing the hypersurface by a parallel hypersurface, and the so-called angle functions of the Gaussian image. The angle functions will be fundamental invariants of Lagrangian submanifolds of $Q^{* n}$. Theorem 4.5 gives an analogue of a formula proven in [12] for the case of $Q^{n}$ in the case of $Q^{* n}$ : it expresses the mean curvature of a Lagrangian submanifold of $Q^{* n}$ in terms of the principal curvatures of a corrsponding hypersurface of $H_{1}^{n+1}(-1)$. We provide several examples of spacelike hypersurfaces of anti-de Sitter space and their Gauss maps to illustrate the theorems.

In the final two sections of the paper we classify two special classes of minimal Lagrangian submanifolds of $Q^{* n}$, namely those with parallel second fundamental form (Theorem 5.1) and those for which the induced sectional curvature is constant (Theorem 6.1). In both cases, the Lagrangian immersions have to be totally geodesic and they correspond to Gauss maps of well-known families of spacelike hypersurfaces of $H_{1}^{n+1}(-1)$.

\section{The GeOMEtry of The COMPLEX HYPERBolic QUADRIC $Q^{* n}$}

We first introduce some notation which will be used throughout the paper. For integers $d$ and $i$ satisfying $0 \leq i \leq d$, the pseudo-Euclidean space of dimension $d$ and index $i$, denoted as $\mathbb{R}_{i}^{d}$, is $\mathbb{R}^{d}$ equipped with the metric $\left\langle\left(x_{1}, \ldots, x_{d}\right),\left(y_{1}, \ldots, y_{d}\right)\right\rangle_{i}=-x_{1} y_{1}-\ldots-x_{i} y_{i}+x_{i+1} y_{i+1}+\ldots+x_{d} y_{d}$. The pseudo-hyperbolic space $H_{i}^{d}(c)$ of dimension $d$, index $i$ and constant sectional curvature $c<0$ is then

$$
H_{i}^{d}(c)=\left\{x \in \mathbb{R}_{i+1}^{d+1} \mid\langle x, x\rangle_{i+1}=\frac{1}{c}\right\} .
$$

In particular, for $i=0$, we have the $d$-dimensional hyperbolic space $H_{0}^{d}(c)=H^{d}(c)$ and for $i=1$, we have the $d$-dimensional anti-de Sitter space $H_{1}^{d}(c)$.

To define the complex hyperbolic quadric, we first consider the complex space $\mathbb{C}_{2}^{n+2}$, which is $\mathbb{C}^{n+2}$ with the metric $\left\langle\left\langle\left(z_{0}, \ldots, z_{n+1}\right),\left(w_{0}, \ldots, w_{n+1}\right)\right\rangle_{2}=\operatorname{Re}\left(-z_{0} \bar{w}_{0}-z_{1} \bar{w}_{1}+\cdots+z_{n+1} \bar{w}_{n+1}\right)\right.$. Remark that, under the natural identification of $\mathbb{C}$ with $\mathbb{R}^{2}$, we have $\mathbb{C}_{2}^{n+2} \approx \mathbb{R}_{4}^{2 n+4}$. We now define the complex anti-de Sitter space $\mathbb{C} H_{1}^{n+1}$ as the set of all complex 1-dimensional subspaces of $\mathbb{C}_{2}^{n+2}$, on which $\left\langle\langle\cdot, \cdot\rangle_{2}\right.$ is negative definite. If we equip $\mathbb{C} H_{1}^{n+1}$ with a natural differential structure, the projection map $\left\{z \in \mathbb{C}_{2}^{n+2} \mid\langle\langle z, z\rangle\rangle_{2}<0\right\} \rightarrow \mathbb{C} H_{1}^{n+1}: z \mapsto[z]$, where $[z]$ denotes the complex line generated by $z$, is a submersion. This remains true if we restrict the map to $H_{3}^{2 n+3}(-1) \subset \mathbb{R}_{4}^{2 n+4}$. (Note that, under the identification $\mathbb{R}_{4}^{2 n+4} \approx \mathbb{C}_{2}^{n+2}$, we have $H_{3}^{2 n+3}(-1) \subset\left\{z \in \mathbb{C}_{2}^{n+2} \mid\langle\langle z, z\rangle\rangle_{2}<0\right\}$.) 
From now on, we refer to the map

$$
\pi: H_{3}^{2 n+3}(-1) \subset \mathbb{C}_{2}^{n+2} \rightarrow \mathbb{C} H_{1}^{n+1}: z \mapsto[z]
$$

as the Hopf fibration. If we equip $\mathbb{C} H_{1}^{n+1}$ with a pseudo-Riemannian metric $g$ such that $\pi$ becomes a pseudo-Riemannian submersion, it turns out that this metric has to have complex index 1 (as already suggested by the notation $\mathbb{C} H_{1}^{n+1}$ ) and constant holomorphic sectional curvature -4 . We denote $\mathbb{C} H_{1}^{n+1}(-4)=\left(\mathbb{C} H_{1}^{n+1}, g\right)$.

Note that the fibers of the Hopf fibration are timelike curves. In fact, for any $z \in H_{3}^{2 n+3}(-1)$, we have that $\pi^{-1}([z])=\left\{e^{i t} z \mid t \in \mathbb{R}\right\}$ and $\operatorname{ker}(d \pi)_{z}=\operatorname{span}\left\{\xi_{z}\right\}$, where $\xi_{z}=i z$ satisfies $\left\langle\left\langle\xi_{z}, \xi_{z}\right\rangle\right\rangle_{2}=$ $\langle\langle z, z\rangle\rangle_{2}=-1$. The complex structure $J$ on $\mathbb{C} H_{1}^{n+1}(-4)$ is induced by multiplication by $i$ on $\mathrm{TH}_{3}^{2 n+3}(-1)$ and $\left(\mathbb{C} H_{1}^{n+1}(-4), g, J\right)$ is a Kähler manifold.

We define the complex hyperbolic quadric of dimension $n$ as

$$
Q^{* n}=\left\{\left[\left(z_{0}, z_{1}, \ldots, z_{n+1}\right)\right] \in \mathbb{C} H_{1}^{n+1}(-4) \mid-z_{0}^{2}-z_{1}^{2}+\cdots+z_{n+1}^{2}=0\right\} .
$$

If $Q^{* n}$ is equipped with the induced metric $\left.g\right|_{Q^{* n}}$, which we will again denote by $g$, and the induced almost complex structure $\left.J\right|_{Q^{* n}}$, which we will again denote by $J$, then $\left(Q^{* n}, g, J\right)$ is a Kähler manifold itself.

The inverse image of $Q^{* n}$ under the Hopf fibration is given by

$$
V_{1}^{* 2 n+1}=\left\{u+i v \mid u, v \in \mathbb{R}_{2}^{n+2},\langle u, u\rangle_{2}=\langle v, v\rangle_{2}=-\frac{1}{2},\langle u, v\rangle_{2}=0\right\} \subset H_{3}^{2 n+3}(-1) .
$$

Remark that $V_{1}^{* 2 n+1}$ is a submanifold of $H_{3}^{2 n+3}(-1)$ of real dimension $2 n+1$. Its real index is 1 , since the normal space to $V_{1}^{* 2 n+1}$ as a submanifold of $H_{3}^{2 n+3}(-1) \subset \mathbb{C}_{2}^{n+2}$ at $z$ is spanned by the orthogonal vectors $\bar{z}$ and $i \bar{z}$, satisfying $\langle\langle\bar{z}, \bar{z}\rangle\rangle_{2}=\langle\langle i \bar{z}, i \bar{z}\rangle\rangle_{2}=-1$. This implies that $Q^{* n}$ is indeed a Riemannian submanifold of $\mathbb{C} H_{1}^{n+1}(-4)$, where the normal space $T_{[z]}^{\perp} Q^{* n}$ is spanned by $(d \pi)_{z}(\bar{z})$ and $J(d \pi)_{z}(\bar{z})=(d \pi)_{z}(i \bar{z})$.

From [10] we have the following.

Lemma 2.1. To any (locally defined) unit normal vector field along $Q^{* n}$ in $\mathbb{C} H_{1}^{n+1}(-4)$, one can associate a field of (locally defined) shape operators. Let $\mathcal{A}$ be the set of all such fields of shape operators. Then an element $A \in \mathcal{A}$ defines a symmetric involutive endomorphism $A_{p}$ of $T_{p} Q^{* n}$, which anti-commutes with $J_{p}$ for every point $p$ of $Q^{* n}$. In particular, $A$ is an almost product structure on (an open subset of) $Q^{* n}$.

If $A \in \mathcal{A}$ is the field of shape operators associated to a unit normal vector field $\zeta$ along $Q^{* n}$ in $\mathbb{C} H_{1}^{n+1}(-4)$, the formula of Weingarten reads

$$
\nabla_{X}^{\mathbb{C} H_{1}^{n+1}(-4)} \zeta=-A X+s(X) J \zeta
$$

for any tangent vector $X$ to $Q^{* n}$, where $\nabla^{\mathbb{C} H_{1}^{n+1}(-4)}$ is the Levi Civita connection of $\mathbb{C} H_{1}^{n+1}(-4)$ and $s$ is a one-form on $Q^{* n}$. Note that this one-form depends on the choice of $\zeta$ and hence on the choice of almost product structure $A$. Using the same reasoning as in $[10,15]$, we obtain

$$
\nabla_{X}^{Q^{* n}} A=s(X) J A
$$

for any tangent vector $X$ to $Q^{* n}$, where $\nabla^{Q^{* n}}$ denotes the Levi-Civita connection on $Q^{* n}$. 
The equation of Gauss for $Q^{* n}$ as a submanifold of $\mathbb{C} H_{1}^{n+1}(-4)$ yields the following expression for the Riemann-Christoffel curvature tensor of $Q^{* n}$ :

$$
\begin{array}{r}
R^{Q^{* n}}(X, Y) Z=-g(Y, Z) X+g(X, Z) Y-g(X, J Z) J Y+g(Y, J Z) J X-2 g(X, J Y) J Z \\
-g(A Y, Z) A X+g(A X, Z) A Y-g(J A Y, Z) J A X+g(J A X, Z) J A Y,
\end{array}
$$

where $A$ is any element of $\mathcal{A}$. In particular, it is an almost product structure on $Q^{* n}$ which anticommutes with $J$ by Lemma 2.1. Notice that the Riemann-Christoffel curvature tensor of $Q^{* n}$ is exactly the negative of the Riemann-Christoffel curvature tensor of $Q^{n}$, which explains why Lagrangian submanifolds of these two spaces can be studied by similar methods, as we will see in the next section.

\section{LAGRANGIAN SUBMANIFOLDS OF $Q^{* n}$}

Consider an immersion $f: M^{n} \rightarrow Q^{* n}$ from a manifold of real dimension $n$ into the complex hyperbolic quadric of complex dimension $n$. If no confusion is possible, we will identify $(d f)_{p}\left(T_{p} M^{n}\right)$ with $T_{p} M^{n}$ for every $p \in M^{n}$. Moreover, we will denote the metric on $M^{n}$, induced from the metric $g$ on $Q^{* n}$, again by $g$. As usual in complex geometry, we say that $f$ is Lagrangian if $J$ maps the tangent space to $M^{n}$ at a point into the normal space to $M^{n}$ at that point and vice versa.

Fixing an almost product structure $A \in \mathcal{A}$ on $Q^{* n}$, we can define, at any point $p$ of a Lagrangian submanifold $M^{n}$ of $Q^{* n}$, two endomorphisms $B$ and $C$ of $T_{p} M^{n}$ by putting

$$
A X=B X-J C X
$$

for all $X \in T_{p} M^{n}$, i.e., $B X$ is the component of $A X$ tangent to $M^{n}$ and $C X$ is the image under $J$ of the component of $A X$ normal to $M^{n}$. The same construction was done in [7] for $Q^{n}$ and, in a similar way, the following result easily follows from Lemma 2.1.

Lemma 3.1. Let $p$ be a point of a Lagrangian submanifold $M^{n}$ of $Q^{* n}$. The endomorphisms $B$ and $C$ of $T_{p} M^{n}$, defined by (3), are symmetric, they commute and they satisfy $B^{2}+C^{2}=\mathrm{id}_{T_{p} M^{n}}$.

Since $Q^{* n}$ is Riemannian, it follows that $B$ and $C$ are simultaneously diagonalizable and the sum of the squares of corresponding eigenvalues is equal to 1 . Thus, there exists an orthonormal basis $\left\{e_{1}, \ldots, e_{n}\right\}$ of $T_{p} M^{n}$ and real numbers $\theta_{1}, \ldots, \theta_{n}$, defined up to an integer multiple of $\pi$, such that $B e_{j}=\cos \left(2 \theta_{j}\right) e_{j}$ and $C e_{j}=\sin \left(2 \theta_{j}\right) e_{j}$ for $j \in\{1, \ldots, n\}$. The factor 2 is just a choice and we can write both equalities together as

$$
A e_{j}=\cos \left(2 \theta_{j}\right) e_{j}-\sin \left(2 \theta_{j}\right) J e_{j} .
$$

Working locally, we can look at $B$ and $C$ as symmetric $(1,1)$-tensor fields on $M^{n}$ which define a local orthonormal frame $\left\{e_{1}, \ldots, e_{n}\right\}$ and local angle functions $\theta_{1}, \ldots, \theta_{n}$.

There are different choices for the almost product structure $A \in \mathcal{A}$ on $Q^{* n}$. The following lemma, whose counterpart for $Q^{n}$ was proven in [7], shows how a change of the almost product structure changes the local angle functions of a Lagrangian submanifold of $Q^{* n}$.

Lemma 3.2. Let $f: M^{n} \rightarrow Q^{* n}$ be a Lagrangian immersion and $A_{0}, A \in \mathcal{A}$. Then there exists a function $\phi: M^{n} \rightarrow \mathbb{R}$ such that, along the image of $f$,

$$
A=\cos \phi A_{0}+\sin \phi J A_{0} .
$$

If $\left\{e_{1}, \ldots, e_{n}\right\}$ is a local orthonormal frame with $A_{0} e_{j}=\cos \left(2 \theta_{j}^{0}\right) e_{j}-\sin \left(2 \theta_{j}^{0}\right) J e_{j}$ for $j \in\{1, \ldots, n\}$, then $A e_{j}=\cos \left(2 \theta_{j}\right) e_{j}-\sin \left(2 \theta_{j}\right) J e_{j}$ for $j \in\{1, \ldots, n\}$, with

$$
\theta_{j}=\theta_{j}^{0}-\frac{\phi}{2}
$$


If $h$ is the second fundamental form of the Lagrangian immersion $f: M^{n} \rightarrow Q^{* n}$, we define

$$
h_{i j}^{k}=g\left(h\left(e_{i}, e_{j}\right), J e_{k}\right)
$$

for all $i, j, k \in\{1, \ldots, n\}$, to be the components of $h$. A fundamental property of Lagrangian submanifolds implies that the components $h_{i j}^{k}$ are symmetric in the three indices. Furthermore, if $\nabla$ is the induced connection on $M^{n}$ from the Levi Civita connection $\nabla^{Q^{* n}}$, we define its connection one-forms by

$$
\omega_{j}^{k}(X)=g\left(\nabla_{X} e_{j}, e_{k}\right)
$$

for all $j, k \in\{1, \ldots, n\}$ and $X$ tangent to $M^{n}$. Remark that these one-forms are anti-symmetric in their indices.

Proposition 3.3. Let $M^{n}$ be a Lagrangian submanifold of $Q^{* n}$ and let $A \in \mathcal{A}$ be an almost product structure on $Q^{* n}$. Let $\left\{e_{1}, \ldots, e_{n}\right\}$ be a local orthonormal frame on $M^{n}$ as constructed above, then the following relations between the angle functions, the components of the second fundamental form and the connection forms hold:

$$
\begin{aligned}
& e_{i}\left(\theta_{j}\right)=h_{j j}^{i}-\frac{s\left(e_{i}\right)}{2}, \\
& \sin \left(\theta_{j}-\theta_{k}\right) \omega_{j}^{k}\left(e_{i}\right)=\cos \left(\theta_{j}-\theta_{k}\right) h_{i j}^{k}
\end{aligned}
$$

for all $i, j, k \in\{1, \ldots, n\}$ with $j \neq k$.

The proof is similar to that of the corresponding result for $Q^{n}$, which can be found in [7]. The following result easily follows from (5).

Corollary 3.4. Let $f: M^{n} \rightarrow Q^{* n}$ be a minimal Lagrangian immersion for which the sum of the local angle functions is constant. Then the one-form $s$ associated to $A$ vanishes on tangent vectors to $M^{n}$.

Proof. We choose $\left\{e_{1}, \ldots, e_{n}\right\}$ and $\theta_{1}, \ldots, \theta_{n}$ as above. Since the sum of the local angle functions is constant, (5) implies

$$
0=e_{i}\left(\theta_{1}+\ldots+\theta_{n}\right)=h_{11}^{i}+\ldots+h_{n n}^{i}-n \frac{s\left(e_{i}\right)}{2}=-n \frac{s\left(e_{i}\right)}{2},
$$

where we used minimality in the last equality. We conclude that $s\left(e_{i}\right)=0$ for all $i \in\{1, \ldots, n\}$, which means that $s$ vanishes on all tangent vectors to $M^{n}$.

We will now state the equations of Gauss and Codazzi for a Lagrangian submanifold of $Q^{* n}$.

Proposition 3.5 (Equations of Gauss and Codazzi). Let $f: M^{n} \rightarrow Q^{* n}$ be a Lagrangian immersion with second fundamental form $h$. Define $B$ and $C$ as above for any choice of $A \in \mathcal{A}$. Finally, denote by $R$ the Riemann-Christoffel curvature tensor of $M^{n}$ and by $\bar{\nabla}$ the connection of Van der Waerden-Bortolotti. Then

$$
\begin{aligned}
g(R(X, Y) Z, W)=- & g(Y, Z) g(X, W)+g(X, Z) g(Y, W) \\
& -g(B Y, Z) g(B X, W)+g(B X, Z) g(B Y, W) \\
& -g(C Y, Z) g(C X, W)+g(C X, Z) g(C Y, W) \\
& +g(h(Y, Z), h(X, W)-g(h(X, Z), h(Y, W))
\end{aligned}
$$

and

$$
\begin{aligned}
(\bar{\nabla} h)(X, Y, Z)-(\bar{\nabla} h)(Y, X, Z)= & g(B Y, Z) J C X-g(B X, Z) J C Y \\
& -g(C Y, Z) J B X+g(C X, Z) J B Y
\end{aligned}
$$


for any vector fields $X, Y, Z$ and $W$ tangent to $M^{n}$.

Proof. These follow immediately from the general forms of the equations of Gauss and Codazzi,

$$
\begin{aligned}
& g(R(X, Y) Z, W)=g\left(R^{Q^{* n}}(X, Y) Z, W\right)+g(h(Y, Z), h(X, W))-g(h(X, Z), h(Y, W)), \\
& (\bar{\nabla} h)(X, Y, Z)-(\bar{\nabla} h)(Y, X, Z)=\left(R^{Q^{* n}}(X, Y) Z\right)^{\perp},
\end{aligned}
$$

where the superscript $\perp$ denotes the component normal to $M^{n}$, by using (2) and (3).

Remark. Note that the Ricci equation for Lagrangian submanifolds of $Q^{* n}$ is equivalent to the Gauss equation.

We finish this section by giving two specific choices of an almost product structure $A \in \mathcal{A}$, adapted to a given Lagrangian submanifold of $Q^{* n}$.

Choice 1 (Choice of $A$ along a Lagrangian submanifold of $Q^{* n}$ such that the sum of the angle functions vanishes). Given a Lagrangian immersion $f: M^{n} \rightarrow Q^{* n}$, one can choose $A \in \mathcal{A}$ such that the associated local angle functions satisfy

$$
\theta_{1}+\cdots+\theta_{n}=0 \bmod \pi \text {. }
$$

Indeed, let $A_{0} \in \mathcal{A}$ be an arbitrary almost product structure with associated local angle functions $\theta_{1}^{0}, \ldots, \theta_{n}^{0}$ and put $\phi=2\left(\theta_{1}^{0}+\cdots+\theta_{n}^{0}\right) / n$. If we choose $A \in \mathcal{A}$ such that $A=\cos \phi A_{0}+\sin \phi J A_{0}$ along the image of $f$, then it follows from Lemma 3.2 that the local angle functions associated to $A$ satisfy (9). Note that this implies that the condition of Corollary 3.4 can always be met by a suitable choice of $A$.

Choice 2 (Choice of $A$ along a Lagrangian submanifold of $Q^{* n}$ with a given horizontal lift). Assume that both a Lagrangian immersion $f: M^{n} \rightarrow Q^{* n}$ and a horizontal lift $\tilde{f}: M^{n} \rightarrow V_{1}^{* 2 n+1}$ of $f$ are given. It follows from [13] that any Lagrangian immersion into $Q^{* n}$ locally allows such a horizontal lift. If $M^{n}$ is simply connected, the horizontal lift can be defined globally. Since the normal space to $V_{1}^{* 2 n+1}$ in $H_{3}^{2 n+3}(-1) \subset \mathbb{C}_{2}^{n+2}$ at a point $z$ is the complex span of $\bar{z}$, one can take $\zeta$, defined by $\zeta_{f(p)}=(d \pi)_{\tilde{f}(p)}(\overline{\tilde{f}(p)})$, as a unit normal vector field to $Q^{* n}$ in $\mathbb{C} H_{1}^{n+1}(-4)$ along the image of $f$. The corresponding shape operators are given by $A X=-(d \pi)_{\tilde{f}(p)}(\tilde{\tilde{X}})$, where $X$ is any vector tangent to $Q^{* n}$ at a point $f(p)$ and $\tilde{X}$ is its horizontal lift to $\tilde{f}(p)$. In the special case that $v$ is tangent to $M^{n}$ at a point $p$, we have

$$
A(d f)_{p} v=-(d \pi)_{\tilde{f}(p)}(d \overline{\tilde{f}})_{p} v
$$

This $A$ can be extended to an element of $\mathcal{A}$, defined in a neighborhood of $f\left(M^{n}\right)$.

\section{A Gauss map for spacelike hypersurfaces of anti-De Sitter space}

4.1. Definition and properties. We define a notion of Gauss map for spacelike hypersurfaces of the anti-de Sitter space $H_{1}^{n+1}(-1)$. The following definition is inspired by the definition of Gauss map of a hypersurface of a sphere, which was given for instance in [12].

Definition 4.1. Let $a: M^{n} \rightarrow H_{1}^{n+1}(-1) \subset \mathbb{R}_{2}^{n+2}$ be a spacelike immersion and denote by $b$ a unit normal vector field along this immersion, tangent to $H_{1}^{n+1}(-1)$. We define the Gauss map of the hypersurface $a$ by

$$
G: M^{n} \rightarrow Q^{* n}: p \mapsto[a(p)+i b(p)]
$$


Note that $G$ indeed takes values in the complex hyperbolic quadric: for every $p \in M^{n}$, we have that $\langle a(p), a(p)\rangle_{2}=\langle b(p), b(p)\rangle_{2}=-1$ and $\langle a(p), b(p)\rangle_{2}=0$, such that $\frac{1}{\sqrt{2}}(a(p)+i b(p)) \in V_{1}^{* 2 n+1}$ by (1). This implies that $[a(p)+i b(p)]=\left[\frac{1}{\sqrt{2}}(a(p)+i b(p))\right]=\pi\left(\frac{1}{\sqrt{2}}(a(p)+i b(p)) \in Q^{* n}\right.$.

In the following, we shall refer to

$$
\widetilde{G}: M^{n} \rightarrow V_{1}^{* 2 n+1}: p \mapsto \frac{1}{\sqrt{2}}(a(p)+i b(p))
$$

as the canonical lift of the Gauss map of a hypersurface $a: M^{n} \rightarrow H_{1}^{n+1}(-1)$ with a fixed unit normal $b$. Using this lift, we can prove that the Gauss map $G$ is Lagrangian.

Lemma 4.2. The Gauss map $G: M^{n} \rightarrow Q^{* n}$ of a spacelike hypersurface $a: M^{n} \rightarrow H_{1}^{n+1}(-1)$ of anti-de Sitter space is a Lagrangian immersion.

Proof. Let $S$ be the shape operator of the immersion $a$ associated to the unit normal vector field $b$ that was used to construct the Gauss map. Denote by $\left\{e_{1}, \ldots, e_{n}\right\}$ a local orthonormal frame of principal directions of $a$ and by $\lambda_{1}, \ldots, \lambda_{n}$ the corresponding principal curvatures such that $S e_{j}=\lambda_{j} e_{j}$ for $j \in\{1, \ldots n\}$. Then $G$ is a Lagrangian immersion since the canonical lift $\widetilde{G}$ satisfies

$$
(d \widetilde{G}) e_{j}=\frac{1}{\sqrt{2}}\left(1-i \lambda_{j}\right) e_{j}
$$

for all $j \in\{1, \ldots, n\}$, which is perpendicular to $i(d \widetilde{G}) e_{k}$ for all $k \in\{1, \ldots, n\}$.

Remark. We can see from $(11)$ that $(d \widetilde{G}) e_{j}$ is orthogonal to $i \widetilde{G}$ for all $j=1, \ldots, n$. This shows that $\widetilde{G}$ is horizontal, meaning that $\widetilde{G}$ is the unique horizontal lift of $G$, up to multiplication with a factor $e^{i t}$ for some constant $t \in \mathbb{R}$.

Furthermore, hypersurfaces of anti-de Sitter space which are parallel to each other have the same Gauss maps.

Lemma 4.3. Let $a: M^{n} \rightarrow H_{1}^{n+1}(-1)$ be an immersion and let $a^{\prime}: M^{n} \rightarrow H_{1}^{n+1}(-1)$ be parallel to $a$, with the same orientation as $a$. Then a and $a^{\prime}$ have the same Gauss maps.

Proof. Denote by $b$ a unit normal vector field to $a$ and by $b^{\prime}$ a unit normal vector field to $a^{\prime}$, both inducing the same orientation on $M^{n}$. Since $a^{\prime}$ is parallel to $a$ we have that

$$
\begin{aligned}
& a^{\prime}(p)=(\cos t) a(p)+(\sin t) b(p), \\
& b^{\prime}(p)=-(\sin t) a(p)+(\cos t) b(p)
\end{aligned}
$$

for some $t \in \mathbb{R}$. We immediately see that

$$
\left[a^{\prime}(p)+i b^{\prime}(p)\right]=[(\cos t-i \sin t) a(p)+(\sin t+i \cos t) b(p)]=\left[e^{-i t}(a(p)+i b(p))\right]=[a(p)+i b(p)] .
$$

4.2. Examples of spacelike hypersurfaces of anti-de Sitter space and their Gauss maps. We now give several families of examples of spacelike hypersurfaces of $H_{1}^{n+1}(-1)$ and their Gauss maps. We also compute the principal curvatures of the hypersurfaces and the angle functions of the Gauss maps.

Example 1. For any real constant $\alpha$, with $\sin \alpha \neq 0$, the immersion

$$
a_{1}: H^{n}(-1) \rightarrow H_{1}^{n+1}(-1): p \mapsto(\cos \alpha,(\sin \alpha) p)
$$


defines a totally umbilical hypersurface of $H_{1}^{n+1}(-1)$. In the following, we will refer to such an immersion as a standard embedding of $H^{n}$ into $H_{1}^{n+1}(-1)$. If we choose $b_{1}(p)=(\sin \alpha,-(\cos \alpha) p)$ as the unit normal vector field, the principal curvatures are $\lambda_{1}=\ldots=\lambda_{n}=\cot \alpha$. The Gauss map of the hypersurface is given by

$$
G_{1}: H^{n}(-1) \rightarrow Q^{* n}: p \mapsto[(\cos \alpha+i \sin \alpha,(\sin \alpha-i \cos \alpha) p)]=[(i, p)] .
$$

Remark that $G_{1}$ is independent of $\alpha$. This can also be seen as a consequence of Lemma 4.3 , since the hypersurfaces in this family are parallel to each other.

Let us compute the local angle functions of $G_{1}$. If we choose $A \in \mathcal{A}$ as in Choice 2 using the horizontal lift $\widetilde{G}_{1}: H^{n}(-1) \rightarrow V_{1}^{* 2 n+1}: p \mapsto \frac{1}{\sqrt{2}}(i, p)$, formula (10) becomes

$$
A\left(d G_{1}\right)_{p} v=-(d \pi)_{\widetilde{G}_{1}(p)}\left(d \overline{\widetilde{G}_{1}}\right)_{p} v=-(d \pi)_{\widetilde{G}_{1}(p)}\left(d \widetilde{G}_{1}\right)_{p} v=-\left(d\left(\pi \circ \widetilde{G}_{1}\right)\right)_{p} v=-\left(d G_{1}\right)_{p} v,
$$

which shows that $B=-\mathrm{id}$ and $C=0$ or, equivalently, $\theta_{1}=\ldots=\theta_{n}=\frac{\pi}{2} \bmod \pi$. For this choice of $A$, the one-form $s$ vanishes on tangent vectors by Corollary 3.4. From Proposition 3.3, we then obtain that $G_{1}$ is totally geodesic. Note that for a general choice of $A \in \mathcal{A}$, we have

$$
\theta_{1}=\ldots=\theta_{n}=\phi \quad \bmod \pi
$$

for a function $\phi: H^{n}(-1) \rightarrow \mathbb{R}$.

Example 2. To describe the second family of examples, we use the map

$$
\psi: \mathbb{R}_{1}^{k+1} \times \mathbb{R}_{1}^{n-k+1} \rightarrow \mathbb{R}_{2}^{n+2}:\left(p_{1}, \ldots, p_{k+1}, q_{1}, \ldots, q_{n-k+1}\right) \mapsto\left(p_{1}, q_{1}, p_{2}, \ldots, p_{k+1}, q_{2}, \ldots, q_{n-k+1}\right) .
$$

For any real constant $\alpha$, with $\cos \alpha \sin \alpha \neq 0$, the map

$$
a_{2}: H^{k}(-1) \times H^{n-k}(-1) \rightarrow H_{1}^{n+1}(-1):(p, q) \mapsto \psi((\cos \alpha) p,(\sin \alpha) q)
$$

is an immersion from a product of hyperbolic spaces into anti-de Sitter space. We will refer to such an immersion as a standard embedding of $H^{k} \times H^{n-k}$ into $H_{1}^{n+1}(-1)$. If we choose the unit normal $b_{2}(p, q)=\psi((\sin \alpha) p,-(\cos \alpha) q)$, then the principal curvatures of the hypersurface are $\lambda_{1}=\ldots=\lambda_{k}=-\tan \alpha$ and $\lambda_{k+1}=\ldots=\lambda_{n}=\cot \alpha$. Moreover, the Gauss map is given by

$$
G_{2}: H^{k}(-1) \times H^{n-k}(-1) \rightarrow Q^{* n}:(p, q) \mapsto[\psi((\cos \alpha+i \sin \alpha) p,(\sin \alpha-i \cos \alpha) q)]=[\psi(i p, q)] .
$$

As in the previous example, the Gauss map is independent of $\alpha$, since all hypersurfaces of this family are parallel to each other.

The angle functions of $G_{2}$ can be computed as in the previous example, using the horizontal lift $\widetilde{G}_{2}: H^{k}(-1) \times H^{n-k}(-1) \rightarrow V_{1}^{* 2 n+1}:(p, q) \mapsto \frac{1}{\sqrt{2}} \psi(i p, q)$ : for a vector $v$ tangent to $H^{k}(-1)$, we find $A\left(d G_{2}\right)_{(p, q)} v=-\left(d G_{2}\right)_{(p, q)} v$, whereas for a vector $w$ tangent to $H^{n-k}(-1)$, we find $A\left(d G_{2}\right)_{(p, q)} w=$ $\left(d G_{2}\right)_{(p, q)} w$. If we choose the orthonormal frame $\left\{e_{1}, \ldots, e_{n}\right\}$ such that $e_{1}, \ldots, e_{k}$ are tangent to $H^{k}(-1)$ and $e_{k+1}, \ldots, e_{n}$ are tangent to $H^{n-k}(-1)$, then $\theta_{1}=\ldots=\theta_{k}=\frac{\pi}{2} \bmod \pi$ and $\theta_{k+1}=\ldots=\theta_{n}=0 \bmod \pi$. As in the previous example, it follows from Corollary 3.4 and Proposition 3.3 that $G_{2}$ is totally geodesic. Note that, with respect to an arbitrary $A \in \mathcal{A}$,

$$
\theta_{1}=\ldots=\theta_{k}=\phi \quad \bmod \pi, \quad \theta_{k+1}=\ldots=\theta_{n}=\phi+\frac{\pi}{2} \bmod \pi
$$

for a function $\phi: H^{k}(-1) \times H^{n-k}(-1) \rightarrow \mathbb{R}$.

The above examples give rise to totally geodesic Lagrangian submanifolds of $Q^{* n}$. In Section 5 we will prove that they are essentially the only totally geodesic Lagrangian submanifolds of $Q^{* n}$ 
and that they are even the only minimal Lagrangian submanifolds of $Q^{* n}$ with parallel second fundamental form.

The following three families of examples are rotation hypersurfaces of $H_{1}^{n+1}(-1)$. Inspired by [1], where rotation hypersurfaces in Riemannian real space forms were defined, eight types of rotation hypersurfaces in semi-Riemannian real space forms were introduced in [11]. When one requires that the rotation hypersurface is spacelike and the ambient space form is $H_{1}^{n+1}(-1)$, there are three types remaining, depending on the signature of the metric on the rotation axis. We start here from a description of these three families which is closer to [1] than to [11].

Example 3. Let $\varphi\left(t_{1}, \ldots, t_{n-1}\right)=\left(\varphi_{1}\left(t_{1}, \ldots, t_{n-1}\right), \ldots, \varphi_{n}\left(t_{1}, \ldots, t_{n-1}\right)\right)$ be an orthogonal parametrisation of $H^{n-1}(-1) \subset \mathbb{R}_{1}^{n}$ and let $I \subset \mathbb{R} \rightarrow H_{1}^{n+1}(-1): s \mapsto(f(s), g(s), 0, \ldots, 0, h(s))$ be a curve parametrized by arc length. This means that the real functions $f, g$ and $h$ satisfy $-f^{2}-g^{2}+h^{2}=-1$ and $-\left(f^{\prime}\right)^{2}-\left(g^{\prime}\right)^{2}+\left(h^{\prime}\right)^{2}=1$. Then

$$
a_{3}\left(s, t_{1}, \ldots, t_{n-1}\right)=\left(f(s), g(s) \varphi\left(t_{1}, \ldots, t_{n-1}\right), h(s)\right)
$$

parametrizes a rotation hypersurface of $H_{1}^{n+1}(-1)$ for which, in the notation of [11], the axis of rotation $\Pi^{2}$ has signature $(1,-1)$. Note that $b_{3}=\left(h g^{\prime}-g h^{\prime},\left(f h^{\prime}-h f^{\prime}\right) \varphi, f g^{\prime}-g f^{\prime}\right)$ defines a normal vector field to the hypersurface in $H_{1}^{n+1}(-1)$ satisfying $\left\langle b_{3}, b_{3}\right\rangle_{2}=-1$. We can now compute the principal curvatures associated to this choice of unit normal using the same technique as in [1]: the coordinate vector fields $\left\{\partial_{s}, \partial_{t_{1}}, \ldots, \partial_{t_{n-1}}\right\}$ form an orthogonal basis of principal vector fields at every point and the corresponding principal curvatures are respectively

$$
\lambda_{1}=\frac{g^{\prime \prime}-g}{\sqrt{1+\left(g^{\prime}\right)^{2}-g^{2}}}, \quad \lambda_{2}=\ldots=\lambda_{n}=-\frac{\sqrt{1+\left(g^{\prime}\right)^{2}-g^{2}}}{g} .
$$

The Gauss map of the hypersurface $a_{3}$, using the normal vector $b_{3}$, is given by

$$
G_{3}=\left[\left(f+i\left(h g^{\prime}-g h^{\prime}\right),\left(g+i\left(f h^{\prime}-h f^{\prime}\right)\right) \varphi, h+i\left(f g^{\prime}-g f^{\prime}\right)\right)\right] .
$$

If we choose $A \in \mathcal{A}$ as in Choice 2, using the canonical lift of $G_{3}$, we obtain from (10) by a straightforward computation that

$$
A\left(d G_{3}\right) \partial_{s}=\frac{\left(g^{\prime \prime}-g\right)^{2}-\left(1-g^{2}+\left(g^{\prime}\right)^{2}\right)}{\left(g^{\prime \prime}-g\right)^{2}+1-g^{2}+\left(g^{\prime}\right)^{2}}\left(d G_{3}\right) \partial_{s}-\frac{2\left(g^{\prime \prime}-g\right) \sqrt{1-g^{2}+\left(g^{\prime}\right)^{2}}}{\left(g^{\prime \prime}-g\right)^{2}+1-g^{2}+\left(g^{\prime}\right)^{2}} J\left(d G_{3}\right) \partial_{s} .
$$

The coefficients on the right hand side are equal to $\cos \left(2 \theta_{1}\right)$ and $-\sin \left(2 \theta_{1}\right)$, so they determine $\theta_{1}$ up to an integer multiple of $\pi$. In particular, we have

$$
\cot \theta_{1}=\frac{1+\cos \left(2 \theta_{1}\right)}{\sin \left(2 \theta_{1}\right)}=\frac{g^{\prime \prime}-g}{\sqrt{1+\left(g^{\prime}\right)^{2}-g^{2}}}=\lambda_{1} .
$$

This is not a coincidence. Theorem 4.4 below states that for the particular choice of $A$ we have made here, one always has $\lambda_{j}=\cot \theta_{j}$. In the present example, we can also compute

$$
A\left(d G_{3}\right) \partial_{t_{i}}=\frac{-2 g^{2}+1+\left(g^{\prime}\right)^{2}}{1+\left(g^{\prime}\right)^{2}}\left(d G_{3}\right) \partial_{t_{i}}+\frac{2 g \sqrt{1-g^{2}+\left(g^{\prime}\right)^{2}}}{1+\left(g^{\prime}\right)^{2}} J\left(d G_{3}\right) \partial_{t_{i}}
$$

for $i \in\{1, \ldots, n-1\}$, from which it follows that

$$
\cot \theta_{j}=-\frac{\sqrt{1+\left(g^{\prime}\right)^{2}-g^{2}}}{g}=\lambda_{j}
$$

for $j \in\{2, \ldots, n\}$. 
Example 4. Let $\varphi\left(t_{1}, \ldots, t_{n-1}\right)=\left(\varphi_{1}\left(t_{1}, \ldots, t_{n-1}\right), \ldots, \varphi_{n}\left(t_{1}, \ldots, t_{n-1}\right)\right)$ be an orthogonal parametrisation of $S^{n-1}(1) \subset \mathbb{R}^{n}$ and let $I \subset \mathbb{R} \rightarrow H_{1}^{n+1}(-1): s \mapsto(f(s), g(s), h(s), 0, \ldots, 0)$ be a curve parametrized by arc length. Hence, the functions $f, g$ and $h$ satisfy $-f^{2}-g^{2}+h^{2}=-1$ and $-\left(f^{\prime}\right)^{2}-\left(g^{\prime}\right)^{2}+\left(h^{\prime}\right)^{2}=1$. Then

$$
a_{4}\left(s, t_{1}, \ldots, t_{n-1}\right)=\left(f(s), g(s), h(s) \varphi\left(t_{1}, \ldots, t_{n-1}\right)\right)
$$

parametrizes a rotation hypersurface of $H_{1}^{n+1}(-1)$ for which, in the notation of [11], the axis of rotation $\Pi^{2}$ has signature $(-1,-1)$. Note that $b_{4}=\left(h g^{\prime}-g h^{\prime}, f h^{\prime}-h f^{\prime},\left(g f^{\prime}-f g^{\prime}\right) \varphi\right)$ is a normal vector field to the hypersurface in $H_{1}^{n+1}(-1)$ for which $\left\langle b_{4}, b_{4}\right\rangle_{2}=-1$. As in the previous example, we can compute the principal curvatures associated to $b_{4}$ to be

$$
\lambda_{1}=\frac{h-h^{\prime \prime}}{\sqrt{\left(h^{\prime}\right)^{2}-h^{2}-1}}, \quad \lambda_{2}=\ldots=\lambda_{n}=\frac{\sqrt{\left(h^{\prime}\right)^{2}-h^{2}-1}}{h} .
$$

Also here, these correspond to the principal vector fields $\partial_{s}, \partial_{t_{1}}, \ldots, \partial_{t_{n-1}}$ respectively.

The Gauss map of the hypersurface $a_{4}$ is given by

$$
G_{4}=\left[\left(f+i\left(h g^{\prime}-g h^{\prime}\right), g+i\left(f h^{\prime}-h f^{\prime}\right),\left(h+i\left(f g^{\prime}-g f^{\prime}\right)\right) \varphi\right)\right] .
$$

Choosing $A \in \mathcal{A}$ as in Choice 2 for the canonical lift of $G_{4}$, we can compute

$$
\begin{aligned}
& A\left(d G_{4}\right) \partial_{s}=\frac{\left(h-h^{\prime \prime}\right)^{2}-\left(\left(h^{\prime}\right)^{2}-h^{2}-1\right)}{\left(h-h^{\prime \prime}\right)^{2}+\left(h^{\prime}\right)^{2}-h^{2}-1}\left(d G_{4}\right) \partial_{s}-\frac{2\left(h-h^{\prime \prime}\right) \sqrt{\left(h^{\prime}\right)^{2}-h^{2}-1}}{\left(h-h^{\prime \prime}\right)^{2}+\left(h^{\prime}\right)^{2}-h^{2}-1} J\left(d G_{4}\right) \partial_{s}, \\
& A\left(d G_{4}\right) \partial_{t_{i}}=\frac{\left(h^{\prime}\right)^{2}-2 h^{2}-1}{\left(h^{\prime}\right)^{2}-1}\left(d G_{4}\right) \partial_{t_{i}}-\frac{2 h \sqrt{\left(h^{\prime}\right)^{2}-h^{2}-1}}{\left(h^{\prime}\right)^{2}-1} J\left(d G_{4}\right) \partial_{t_{i}}
\end{aligned}
$$

for $i \in\{1, \ldots, n-1\}$. As in the previous example, we obtain

$$
\cot \theta_{1}=\frac{h-h^{\prime \prime}}{\sqrt{\left(h^{\prime}\right)^{2}-h^{2}-1}}=\lambda_{1}, \quad \cot \theta_{j}=\frac{\sqrt{\left(h^{\prime}\right)^{2}-h^{2}-1}}{h}=\lambda_{j}
$$

for $j \in\{2, \ldots, n\}$.

Example 5. To describe the last example, we will use a different basis of $\mathbb{R}_{2}^{n+2}$. Denoting the standard basis by $\left\{e_{1}, \ldots, e_{n+2}\right\}$, we define $u_{1}=e_{1}, u_{2}=e_{3}+e_{2}, u_{3}=e_{3}-e_{2}$ and $u_{j}=e_{j}$ for $j \in\{4, \ldots, n+2\}$. Note that $u_{2}$ and $u_{3}$ are null vectors satisfying $\left\langle u_{2}, u_{3}\right\rangle_{2}=2$. Consider a curve $I \subset \mathbb{R} \rightarrow H_{1}^{n+1}(-1): s \mapsto f(s) u_{1}+g(s) u_{2}+h(s) u_{3}$ parametrized by arc length. This means that the functions $f, g$ and $h$ satisfy $-f^{2}+4 g h=-1$ and $-\left(f^{\prime}\right)^{2}+4 g^{\prime} h^{\prime}=1$. Then

$a_{5}\left(s, t_{1}, \ldots, t_{n-1}\right)=f(s) u_{1}+\frac{f^{2}(s)-1-h^{2}(s) \sum_{i=1}^{n-1} t_{i}^{2}}{4 h} u_{2}+h(s) u_{3}+h(s) t_{1} u_{4}+\ldots+h(s) t_{n-1} u_{n+2}$ parametrizes a rotation hypersurface of $H_{1}^{n+1}(-1)$ for which, in the notation of [11], the axis of rotation $\Pi^{2}$ has signature $(-1,0)$. We define the following vector field :

$$
\begin{array}{r}
b_{5}=-\sqrt{\left(h^{\prime}\right)^{2}-h^{2}}\left(\frac{h^{\prime} f^{\prime}-h f}{\left(h^{\prime}\right)^{2}-h^{2}} u_{1}+\frac{\left.2 h h^{\prime} f f^{\prime}+\left(\left(h^{\prime}\right)^{2}-h^{2}\right)\left(1+f^{2}\right)-h^{2}\left(\left(h^{\prime}\right)^{2}-h^{2}\right) \sum_{i=1}^{n-1} t_{i}^{2}\right)}{4 h^{2}\left(\left(h^{\prime}\right)^{2}-h^{2}\right)} u_{2}\right. \\
\left.+u_{3}+t_{1} u_{4}+\ldots+t_{n-1} u_{n+2}\right) .
\end{array}
$$


Using that $\left(h f^{\prime}-f h^{\prime}\right)^{2}=\left(h^{\prime}\right)^{2}-h^{2}$, one can check that this is a normal vector field to the hypersurface, satisfying $\left\langle b_{5}, b_{5}\right\rangle_{2}=-1$. The associated principal curvatures are

$$
\lambda_{1}=\frac{h+h^{\prime \prime}}{\sqrt{\left(h^{\prime}\right)^{2}-h^{2}}}, \quad \lambda_{2}=\ldots=\lambda_{n}=\frac{\sqrt{\left(h^{\prime}\right)^{2}-h^{2}}}{h} .
$$

As in the previous examples, these correspond to the principal vector fields $\partial_{s}, \partial_{t_{1}}, \ldots, \partial_{t_{n-1}}$ respectively.

The Gauss map of the hypersurface $a_{5}$ is given by $G_{5}=\left[\left(a_{5}+i b_{5}\right)\right]$. If we choose $A \in \mathcal{A}$ as in Choice 2 for the canonical lift of $G_{5}$, we can compute

$$
\begin{aligned}
& A\left(d G_{5}\right) \partial_{s}=\frac{\left(h+h^{\prime \prime}\right)^{2}-\left(h^{\prime}\right)^{2}+h^{2}}{\left(h+h^{\prime \prime}\right)^{2}+\left(h^{\prime}\right)^{2}-h^{2}}\left(d G_{5}\right) \partial_{s}-\frac{2\left(h+h^{\prime \prime}\right) \sqrt{\left(h^{\prime}\right)^{2}-h^{2}}}{\left(h^{\prime}\right)^{2}-h^{2}+\left(h+h^{\prime \prime}\right)^{2}} J\left(d G_{5}\right) \partial_{s}, \\
& A\left(d G_{5}\right) \partial_{t_{i}}=\frac{\left(h^{\prime}\right)^{2}-2 h^{2}}{\left(h^{\prime}\right)^{2}}\left(d G_{5}\right) \partial_{t_{i}}-\frac{2 h \sqrt{\left(h^{\prime}\right)^{2}-h^{2}}}{\left(h^{\prime}\right)^{2}} J\left(d G_{5}\right) \partial_{t_{i}}
\end{aligned}
$$

for $i \in\{1, \ldots, n-1\}$. As in the previous examples, we find

$$
\cot \theta_{1}=\frac{h+h^{\prime \prime}}{\sqrt{\left(h^{\prime}\right)^{2}-h^{2}}}=\lambda_{1}, \quad \cot \theta_{j}=\frac{\sqrt{\left(h^{\prime}\right)^{2}-h^{2}}}{h}=\lambda_{j}
$$

for $j \in\{2, \ldots, n\}$.

4.3. Relation between principal curvatures and the angle functions of the Gauss map. Knowing that the Gauss map of a hypersurface of $H_{1}^{n+1}(-1)$ is a Lagrangian immersion into $Q^{* n}$ raises the question whether, given a Lagrangian immersion $f: M^{n} \rightarrow Q^{* n}$, we can go back and find a spacelike hypersurface of $H_{1}^{n+1}(-1)$ with Gauss map $f$. It turns out that we can always do this, at least locally. A similar question for Lagrangian immersions into $Q^{n}$ and hypersurfaces of $S^{n}(1)$ was answered in [17], and we can prove the following theorem using similar methods.

Theorem 4.4. Let $a: M^{n} \rightarrow H_{1}^{n+1}(-1)$ be a spacelike hypersurface with unit normal $b$. Then the Gauss map $G: M^{n} \rightarrow Q^{* n}: p \mapsto[a(p)+i b(p)]$ is a Lagrangian immersion. Moreover, if $A$ is chosen as in Choice 2 using the canonical horizontal lift

$$
\widetilde{G}: M^{n} \rightarrow V_{1}^{* 2 n+1}: p \mapsto \frac{1}{\sqrt{2}}(a(p)+i b(p)),
$$

then the relation between the principal curvatures $\lambda_{1}, \ldots, \lambda_{n}$ of a, with respect to the shape operator associated to $b$, and the angle functions $\theta_{1}, \ldots, \theta_{n}$ of $G$ is

$$
\lambda_{j}=\cot \theta_{j}
$$

for $j=1, \ldots, n$.

Conversely, if $f: M^{n} \rightarrow Q^{* n}$ is a Lagrangian immersion, then for every point of $M^{n}$ there exist an open neighborhood $U$ of that point in $M^{n}$ and an immersion $a: U \rightarrow H_{1}^{n+1}(-1)$ with Gauss map $\left.f\right|_{U}$. This immersion is not unique, nor are its principal curvature functions. However, for any choice of a, a local frame of principal directions for a is adapted to $f$ in the sense that (4) holds for any choice of $A$ and the principal curvature functions $\lambda_{1}, \ldots, \lambda_{n}$ of a are related to the corresponding local angle functions $\theta_{1}, \ldots, \theta_{n}$ by

$$
\cot \left(\theta_{j}-\theta_{k}\right)= \pm \frac{\lambda_{j} \lambda_{k}+1}{\lambda_{j}-\lambda_{k}}
$$

for $j, k=1, \ldots, n$ at points where $\lambda_{j} \neq \lambda_{k}$. 
Proof. Let $\left\{e_{1}, \ldots, e_{n}\right\}$ be a local orthonormal frame of principal directions on $M^{n}$ for the immersion $a: M^{n} \rightarrow H_{1}^{n+1}(-1)$. As in [17], one can compute $A(d G) e_{j}$ explicitly, proving the relation (14).

For the converse, consider a Lagrangian immersion $f: M^{n} \rightarrow Q^{* n}$ and fix a point $p \in M^{n}$. By [13], there exists a horizontal lift $\tilde{f}_{0}: U \rightarrow V_{1}^{* 2 n+1}$ of $\left.f\right|_{U}$ for every simply connected open neighborhood $U$ of $p$. Any other horizontal lift of $\left.f\right|_{U}$ can be written as $\tilde{f}_{t}=e^{i t} \tilde{f}_{0}$ for some constant $t \in \mathbb{R}$. Now define $a_{t}=\sqrt{2} \operatorname{Re} \tilde{f}_{t}$ and $b_{t}=\sqrt{2} \operatorname{Im} \tilde{f}_{t}$. If $a_{t}$ is an immersion, it defines a hypersurface of $H_{1}^{n+1}(-1)$ with Gauss map $\left.f\right|_{U}$. To see when this is the case, one can compute $d a_{t}$ in the same way as in [17], concluding that there are infinitely many choices for $t \in \mathbb{R}$ such that $a_{t}$ is an immersion in a neighborhood of $p$. Note that different values of $t$ correspond to parallel hypersurfaces, which have the same Gauss maps. Finally, one can compute $d b_{t}$ to find a relation between the principal curvatures of $a_{t}$ and the local angle functions of $\left.f\right|_{U}$. The same computation as in [17] yields that the difference of two local angle functions does not depend on the choice of $A \in \mathcal{A}$ and is related to the principal curvatures by (15).

Let us revisit the examples given above. By comparing the principal curvatures and angle functions given in all the examples, we see that the relation (15) is still valid if we allow the value $\infty$. Note also that (15) holds for any two chosen indices. In Example 3, Example 4 and Example 5 we have chosen $A \in \mathcal{A}$ as in Choice 2 for the canonical horizontal lift of the Gauss map and we noticed already that (14) holds.

4.4. Relation between principal curvatures and the mean curvature of the Gauss map. There is also a relation between the principal curvatures of a spacelike hypersurface of anti-de Sitter space and the mean curvature of its Gauss map, which is given by the following theorem. A similar result for hypersurfaces of $S^{n+1}(1)$ and their Gauss maps into $Q^{n}$ was proven in [12]. Here we give a slightly different proof, but the main ideas are similar.

Theorem 4.5. Let $a: M^{n} \rightarrow H_{1}^{n+1}(-1)$ be an immersion and let $G: M^{n} \rightarrow Q^{* n}$ be the Gauss map of this immersion. Denote by $\lambda_{1}, \ldots, \lambda_{n}$ the eigenvalues of the shape operator of a, associated to the unit normal $b$ that was used to construct $G$. If $H$ is the mean curvature vector of $G$, then

$$
g(J H, \cdot)=\frac{1}{n} d\left(\sum_{i=1}^{n} \arctan \left(\lambda_{j}\right)\right)=\frac{1}{n} d\left(\operatorname{Im}\left(\log \left(\prod_{j=1}^{n}\left(1+i \lambda_{j}\right)\right)\right)\right) .
$$

Proof. Let $\left\{\epsilon_{1}, \ldots, \epsilon_{n}\right\}$ be an orthonormal frame on $M^{n}$ with respect to the metric induced by $a$, such that $S \epsilon_{j}=\lambda_{j} \epsilon_{j}$ for $j \in\{1, \ldots, n\}$, where $S$ is the shape operator associated to $b$. Then the vector fields $e_{j}=\frac{\sqrt{2}}{\sqrt{1+\lambda_{j}^{2}}} \epsilon_{j}$ form an orthonormal frame on $M^{n}$ with respect to the metric induced by the canonical lift $\tilde{G}$. If $D$ is the Euclidean connection on $\mathbb{R}_{2}^{2 n+4}$, we can identify $e_{j}$ with $(d \widetilde{G}) e_{j}=D_{e_{j}} \tilde{G}$, its image under the derivative of $\tilde{G}$.

We compute $g(J H, X)$ for a tangent vector $X$ to $M^{n}$. Since $n H$ is the trace of the second fundamental form $h$ and $g(h(\cdot, \cdot), J \cdot)$ is totally symmetric, it follows that

$$
n g(J H, X)=\sum_{j=1}^{n} g\left(J h\left(e_{j}, e_{j}\right), X\right)=-\sum_{j=1}^{n} g\left(\nabla_{X}^{Q^{* n}} e_{j}, J e_{j}\right)=-\sum_{j=1}^{n}\left\langle\left\langle D_{X} D_{e_{j}} \tilde{G}, i D_{e_{j}} \tilde{G}\right\rangle\right\rangle_{2} .
$$


Since $e_{j}=\frac{\sqrt{2}}{\sqrt{1+\lambda_{j}^{2}}} \epsilon_{j}$, we get from (11) that

$$
\begin{array}{r}
\left\langle\left\langle D_{X} D_{e_{j}} \tilde{G}, i D_{e_{j}} \tilde{G}\right\rangle\right\rangle_{2}=\frac{2}{1+\lambda_{j}^{2}}\left\langle\left\langle D_{X} D_{\epsilon_{j}} \tilde{G}, i D_{\epsilon_{j}} \tilde{G}\right\rangle\right\rangle_{2}=\frac{1}{1+\lambda_{j}^{2}}\left\langle\left\langle D_{X}\left(1-i \lambda_{j}\right) \epsilon_{j},\left(i+\lambda_{j}\right) \epsilon_{j}\right\rangle\right\rangle_{2} \\
=\frac{1}{1+\lambda_{j}^{2}}\left\langle\left\langle-i X\left(\lambda_{j}\right) \epsilon_{j}, i \epsilon_{j}\right\rangle\right\rangle_{2}=-\frac{X\left(\lambda_{j}\right)}{1+\lambda_{j}^{2}}=-X\left(\arctan \left(\lambda_{j}\right)\right)=-X\left(\operatorname{Im}\left(\log \left(1+i \lambda_{j}\right)\right)\right) .
\end{array}
$$

The result follows by substituting this in (17).

The Gauss maps $G_{1}$ and $G_{2}$ are minimal immersions since the principal curvatures of the corresponding hypersurfaces of anti-de Sitter space are constant.

\section{Minimal Lagrangian submanifolds of $Q^{* n}$ With Parallel SECOND fundamental FORM}

In this section we classify all minimal Lagrangian submanifolds of $Q^{* n}$ with parallel second fundamental form. In particular, we show that they are totally geodesic and essentially correspond to the Gauss maps from Example 1 and Example 2 above.

Theorem 5.1. Let $f: M^{n} \rightarrow Q^{* n}$, with $n \geq 2$, be a minimal Lagrangian immersion with parallel second fundamental form. Then, up to isometries of $Q^{* n}, f$ is the Gauss map of a standard embedding of an open part of $H^{n}$ or of $H^{k} \times H^{n-k}$ into $H_{1}^{n+1}(-1)$. In particular, $f$ is totally geodesic.

Proof. Since $\bar{\nabla} h=0$, the equation of Codazzi (8) yields

$$
g(B Y, Z) C X-g(B X, Z) C Y-g(C Y, Z) B X+g(C X, Z) B Y=0
$$

for all vector fields $X, Y$ and $Z$ on $M^{n}$. Denote by $\left\{e_{1}, \ldots, e_{n}\right\}$ a local orthonormal frame on $M^{n}$, diagonalizing $B$ and $C$ and corresponding to local angle functions $\theta_{1}, \ldots, \theta_{n}$. By choosing $X=Z=e_{i}$ and $Y=e_{j}$ for different indices $i$ and $j$, we obtain $\sin \left(2\left(\theta_{i}-\theta_{j}\right)\right)=0$ and hence

$$
\forall i, j \in\{1, \ldots, n\}, \exists m_{i j} \in \mathbb{Z}: \theta_{i}-\theta_{j}=m_{i j} \frac{\pi}{2} .
$$

It now follows from Theorem 4.4 that $f$ is the Gauss map of an immersion $a: M^{n} \rightarrow H_{1}^{n+1}(-1)$ whose principal curvature functions $\lambda_{1}, \ldots, \lambda_{n}$ satisfy

$$
\forall i, j \in\{1, \ldots, n\}: \lambda_{i}=\lambda_{j} \text { or } \lambda_{i} \lambda_{j}+1=0 .
$$

On the other hand, the equation of Codazzi for the immersion $a$ yields that

$$
\forall i, j \in\{1, \ldots, n\}: e_{i}\left(\lambda_{j}\right) e_{j}-e_{j}\left(\lambda_{i}\right) e_{i}=0 .
$$

It follows from (18) that there are at most two different principal curvatures and, if there are two different ones, their product is equal to -1 . Combining this with (19) yields that the principal curvatures are constant, i.e., that the hypersurface $a$ is isoparametric. It was proven in [14] that there are only two types of spacelike isoparametric hypersurfaces of anti-de Sitter space and it is not hard to see that these two families precisely correspond to Example 1 and Example 2. We already checked that the Gauss maps in these examples are totally geodesic.

Corollary 5.2. Let $f: M^{n} \rightarrow Q^{* n}$, with $n \geq 2$, be a totally geodesic Lagrangian immersion. Then, up to isometries of $Q^{* n}, f$ is the Gauss map of a standard embedding of an open part of $H^{n}$ or of $H^{k} \times H^{n-k}$ into $H_{1}^{n+1}(-1)$. 


\section{Minimal Lagrangian submanifolds of $Q^{* n}$ With Constant Sectional CuRVAture}

We can compute the sectional curvature of a Lagrangian submanifold of $Q^{* n}$ from the equation of Gauss (7). If $f: M^{n} \rightarrow Q^{* n}$ is a Lagrangian immersion and $\left\{e_{1}, \ldots, e_{n}\right\}$ is a local orthonormal frame on $M^{n}$ such that $A e_{i}=\cos \left(2 \theta_{i}\right) e_{i}-\sin \left(2 \theta_{i}\right) J e_{i}$ for some $A \in \mathcal{A}$ and all $i \in\{1, \ldots, n\}$, then the sectional curvature of the metric on $M^{n}$ induced by $f$ is determined by

$$
K\left(\operatorname{span}\left\{e_{i}, e_{j}\right\}\right)=-2 \cos ^{2}\left(\theta_{i}-\theta_{j}\right)+g\left(h\left(e_{i}, e_{i}\right), h\left(e_{j}, e_{j}\right)\right)-g\left(h\left(e_{i}, e_{j}\right), h\left(e_{i}, e_{j}\right)\right) .
$$

In particular, the Gauss map $G_{1}$ from Example 1 gives rise to a metric of constant sectional curvature $c=-2$ on $H^{n}(-1)$ and, in dimension $n=2$, the Gauss map $G_{2}$ from Example 2 gives rise to a metric of constant sectional curvature $c=0$ on $H^{1}(-1) \times H^{1}(-1)$.

In this section we prove that these two are essentially the only minimal Lagrangian submanifolds with constant sectional curvature of $Q^{* n}$.

Theorem 6.1. Let $f: M^{n} \rightarrow Q^{* n}, n \geq 2$, be a minimal Lagrangian immersion such that $M^{n}$ has constant sectional curvature c. Then, up to isometries of $Q^{* n}$, $f$ is the Gauss map of a standard embedding of an open part of $H^{n}$ into $H_{1}^{n+1}(-1)$ or of $H^{1} \times H^{1}$ into $H_{1}^{3}(-1)$. In the former case $c=-2$ and in the latter case $c=0$.

The proof of the theorem relies on several lemmas and propositions. We will only briefly indicate how to adjust the proofs from their counterparts for minimal Lagrangian submanifolds with constant sectional curvature of $Q^{n}$, see [7].

Lemma 6.2. Let $f: M^{n} \rightarrow Q^{* n}$ be a Lagrangian immersion such that $M^{n}$ has constant sectional curvature. Assume an almost complex structure $A \in \mathcal{A}$ is fixed on $Q^{* n}$ and $\left\{e_{1}, \ldots, e_{n}\right\}$ is an orthonormal frame on $M^{n}$ such that $A e_{i}=\cos \left(2 \theta_{i}\right) e_{i}-\sin \left(2 \theta_{i}\right) J e_{i}$ for all $i \in\{1, \ldots, n\}$. Then

$$
\begin{aligned}
& \sin \left(\theta_{i}-\theta_{k}\right) \sin \left(2 \theta_{j}-\theta_{k}-\theta_{i}\right)\left(h_{i \ell}^{k} J e_{j}+\delta_{j \ell} h\left(e_{i}, e_{k}\right)\right) \\
& \quad+\sin \left(\theta_{k}-\theta_{j}\right) \sin \left(2 \theta_{i}-\theta_{j}-\theta_{k}\right)\left(h_{j \ell}^{k} J e_{i}+\delta_{i \ell} h\left(e_{k}, e_{j}\right)\right) \\
& \quad+\sin \left(\theta_{j}-\theta_{i}\right) \sin \left(2 \theta_{k}-\theta_{i}-\theta_{j}\right)\left(h_{i \ell}^{j} J e_{k}+\delta_{k \ell} h\left(e_{j}, e_{i}\right)\right)=0
\end{aligned}
$$

for all $i, j, k, l \in\{1, \ldots, n\}$. In particular,

$$
\begin{aligned}
& h_{i i}^{k} \sin \left(\theta_{k}-\theta_{i}\right) \sin \left(\theta_{i}+\theta_{k}-2 \theta_{j}\right)=h_{j j}^{k} \sin \left(\theta_{k}-\theta_{j}\right) \sin \left(\theta_{j}+\theta_{k}-2 \theta_{i}\right), \\
& h_{i j}^{k} \sin \left(\theta_{j}-\theta_{i}\right) \sin \left(\theta_{i}+\theta_{j}-2 \theta_{k}\right)=0
\end{aligned}
$$

for mutually different $i, j, k \in\{1, \ldots, n\}$ and

$$
h_{i j}^{k} \sin \left(\theta_{i}-\theta_{j}\right) \sin \left(\theta_{i}+\theta_{j}-2 \theta_{\ell}\right)=0
$$

for mutually different $i, j, k, l=1, \ldots, n$.

Proof. To modify the proof of the corresponding result in [7], take $T$ to be the $(1,3)$-tensor field given by $T(X, Y, Z)=g(C X, Z) J B Y-g(C Y, Z) J B X-g(B X, Z) J C Y+g(B Y, Z) J C X$.

Lemma 6.3. For $n \geq 3$, let $f: M^{n} \rightarrow Q^{* n}$ be a minimal Lagrangian immersion such that $M^{n}$ has constant sectional curvature. Then the local angle functions are either all equal or all pairwise unequal (as functions) modulo $\pi$. In the former case, the immersion is the Gauss map of a standard embedding of an open part of $H^{n}$ into $H_{1}^{n+1}(-1)$. 
Proof. To modify the proof of the corresponding result in [7], notice that the sectional curvature $K$ of $M^{n}$ satisfies $K\left(\operatorname{span}\left\{e_{i}, e_{j}\right\}\right)=-2 \cos ^{2}\left(\theta_{i}-\theta_{j}\right)+g\left(h\left(e_{i}, e_{i}\right), h\left(e_{j}, e_{j}\right)\right)-g\left(h\left(e_{i}, e_{j}\right), h\left(e_{i}, e_{j}\right)\right)$, where $\left\{e_{1}, \ldots, e_{n}\right\}$ is an orthonormal frame on $M^{n}$ such that $A e_{i}=\cos \left(2 \theta_{i}\right) e_{i}-\sin \left(2 \theta_{i}\right) J e_{i}$ for all $i \in\{1, \ldots, n\}$.

Lemma 6.4. Let $f: M^{n} \rightarrow Q^{* n}$ be a minimal Lagrangian immersion such that $M^{n}$ has constant sectional curvature. Then $h_{i j}^{k}=0$ for all mutually different indices $i, j$ and $k$.

Proof. Because of Lemma 6.2 and 6.3 we can use the same argumentation as in [7].

Theorem 6.1 is proven by considering the dimensions $n=2, n=3, n=4$ and $n \geq 5$ separately. The only differences with respect to the situation of $Q^{n}$ occur for $n=2$ and $n=3$, so we will prove Propositions 6.5 and 6.6 below, but not Proposition 6.7 (which corresponds to two propositions in [7]: one for $n=4$ and one for $n \geq 5$ ).

\subsection{Classification in dimension $n=2$.}

Proposition 6.5. If $f: M^{2} \rightarrow Q^{* 2}$ is a minimal Lagrangian immersion such that the induced metric on $M^{2}$ has constant Gaussian curvature, then either the induced metric has Gaussian curvature -2 and $f$ is the Gauss map of a part of a standard embedding $H^{2} \rightarrow H_{1}^{3}(-1)$, or the induced metric is flat and $f$ is the Gauss map of a part of a standard embedding $H^{1} \times H^{1} \rightarrow H_{1}^{3}(-1)$.

Proof. Since $Q^{* 2}$ is isometric to $H^{2}(-4) \times H^{2}(-4)$, the result follows from the classification of minimal Lagrangian surfaces with constant Gaussian curvature in $H^{2}(c) \times H^{2}(c)$, given in [2].

\subsection{Classification in dimension $n=3$.}

Proposition 6.6. Let $f: M^{3} \rightarrow Q^{* 3}$ be a Lagrangian minimal immersion with constant sectional curvature, then $M^{3}$ has constant sectional curvature -2 and $f$ is the Gauss map of part of a standard embedding $H^{3}(c) \rightarrow H_{1}^{4}(-1)$.

Proof. Choose $A \in \mathcal{A}$ as in Choice 1. From Lemma 6.3 and Lemma 6.4, it follows that we only need to consider the case where all local angle functions are different modulo $\pi$ and $h_{12}^{3}=0$.

We denote

$$
\begin{aligned}
& x=\sin \left(\theta_{1}-\theta_{2}\right) \sin \left(\theta_{1}+\theta_{2}-2 \theta_{3}\right), \\
& y=\sin \left(\theta_{2}-\theta_{3}\right) \sin \left(\theta_{2}+\theta_{3}-2 \theta_{1}\right), \\
& z=\sin \left(\theta_{3}-\theta_{1}\right) \sin \left(\theta_{3}+\theta_{1}-2 \theta_{2}\right) .
\end{aligned}
$$

It follows from simple trigonometric identities that $x+y+z=0$. Moreover, (21) is equivalent to

$$
h_{22}^{1} x+h_{33}^{1} z=0, \quad h_{11}^{2} x+h_{33}^{2} y=0, \quad h_{11}^{3} z+h_{22}^{3} y=0 .
$$

We will distinguish three cases according to the function values of $x, y$ and $z$ at a single, arbitrarily chosen point.

Case 1: At least two of the local functions $x, y$ and $z$ are zero. In this case all 3 must vanish since $x+y+z=0$. Since the local angle functions are mutually different modulo $\pi$, we may assume $\theta_{1}<\theta_{2}<\theta_{3}$. It follows from $x=y=z=0$ that $3 \theta_{1}=3 \theta_{2}=3 \theta_{3} \bmod \pi$. Therefore, $\theta_{1}, \theta_{2}$ and $\theta_{3}$ are all constant. This implies that the principal curvatures of the corresponding spacelike hypersurface in anti-de Sitter are also constant, which contradicts the result of [14].

Case 2: Exactly one of the local functions $x, y$ and $z$ is zero. Without loss of generality, we may assume that $x=0$, and $y=-z \neq 0$. In follows from (22) that $h_{33}^{1}=h_{33}^{2}=0$ and $h_{11}^{3}=h_{22}^{3}$. 
Since the angle functions are all different, the assumption $x=0$ implies that $\theta_{1}+\theta_{2}-2 \theta_{3}=0$ $\bmod \pi$. Deriving this expression and using (5) gives $h_{11}^{i}+h_{22}^{i}-2 h_{33}^{i}=0$ for all $i \in\{1,2,3\}$. By the minimality condition, we also have that $h_{11}^{i}+h_{22}^{i}+h_{33}^{i}=0$, thus we get $h_{33}^{i}=0$ and $h_{11}^{i}+h_{22}^{i}=0$ for all $i \in\{1,2,3\}$. Therefore, the only possibly non-zero components of the second fundamental form are $h_{11}^{1}=-h_{22}^{1}$ and $h_{11}^{2}=-h_{22}^{2}$. By (20), the sectional curvatures of the planes spanned by $\left\{e_{1}, e_{2}\right\},\left\{e_{1}, e_{3}\right\}$ and $\left\{e_{2}, e_{3}\right\}$ are $K_{12}=-2 \cos ^{2}\left(\theta_{2}-\theta_{1}\right)-2\left(h_{11}^{1}\right)^{2}-2\left(h_{22}^{2}\right)^{2}, K_{13}=-2 \cos ^{2}\left(\theta_{3}-\theta_{1}\right)$ and $K_{23}=-2 \cos \left(\theta_{3}-\theta_{2}\right)$. It follows from the latter two that $\theta_{3}-\theta_{1}$ and $\theta_{3}-\theta_{2}$ are constant. Deriving this and using (5) gives $h_{11}^{i}=0$ and $h_{22}^{i}=0$. We conclude that the submanifold is totally geodesic, but it follows from $K_{12}=K_{13}$ that $y=0$, contradicting the assumption.

Case 3: None of the local functions $x, y$ and $z$ are zero. We work on an open subset of $M^{n}$ where none of the functions vanish. It follows from (21) and the minimality condition that there are local functions $\alpha_{1}, \alpha_{2}$ and $\alpha_{3}$ on this subset such that

$$
\begin{array}{lll}
h_{11}^{1}=\alpha_{1}(z-x), & h_{11}^{2}=-\alpha_{2} y, & h_{11}^{3}=-\alpha_{3} y, \\
h_{22}^{1}=-\alpha_{1} z, & h_{22}^{2}=\alpha_{2}(y-x), & h_{22}^{3}=\alpha_{3} z, \\
h_{33}^{1}=\alpha_{1} x, & h_{33}^{2}=\alpha_{2} x, & h_{33}^{3}=\alpha_{3}(y-z) .
\end{array}
$$

By Corollary 3.4 and equation (5) we find the derivatives of the angle functions. Substituting these in the Codazzi equation (8) for $(X, Y, Z)=\left(e_{1}, e_{2}, e_{3}\right)$ and using $\theta_{1}+\theta_{2}+\theta_{3}=0 \bmod \pi$ to eliminate $\theta_{3}$, we obtain

$$
\begin{gathered}
e_{1}\left(\alpha_{3}\right)=\frac{1}{4} \alpha_{1} \alpha_{3} \csc \left(2 \theta_{1}+\theta_{2}\right)\left(\cos \left(4 \theta_{1}+5 \theta_{2}\right)+7 \cos \left(3 \theta_{2}\right)-5 \cos \left(2 \theta_{1}+\theta_{2}\right)\right. \\
\left.\quad-\cos \left(3\left(2 \theta_{1}+\theta_{2}\right)\right)-2 \cos \left(4 \theta_{1}-\theta_{2}\right)\right) \\
e_{2}\left(\alpha_{3}\right)=\frac{1}{4} \alpha_{2} \alpha_{3} \csc \left(\theta_{1}+2 \theta_{2}\right)\left(-\cos \left(5 \theta_{1}+4 \theta_{2}\right)-7 \cos \left(3 \theta_{1}\right)+5 \cos \left(\theta_{1}+2 \theta_{2}\right)\right. \\
\left.+\cos \left(3\left(\theta_{1}+2 \theta_{2}\right)\right)+2 \cos \left(\theta_{1}-4 \theta_{2}\right)\right)
\end{gathered}
$$

Similarly, for $(X, Y, Z)=\left(e_{2}, e_{3}, e_{1}\right)$ we obtain

$$
\begin{aligned}
e_{3}\left(\alpha_{1}\right)= & \frac{1}{4} \alpha_{1} \alpha_{3} \csc \left(2 \theta_{1}+\theta_{2}\right)\left(-\cos \left(4 \theta_{1}-\theta_{2}\right)-7 \cos \left(3 \theta_{2}\right)+5 \cos \left(2 \theta_{1}+\theta_{2}\right)\right. \\
& \left.\quad+\cos \left(6 \theta_{1}+3 \theta_{2}\right)+2 \cos \left(4 \theta_{1}+5 \theta_{2}\right)\right) \\
e_{2}\left(\alpha_{1}\right)=- & \frac{1}{4} \alpha_{1} \alpha_{2} \csc \left(\theta_{1}-\theta_{2}\right)\left(\cos \left(3 \theta_{1}-3 \theta_{2}\right)-7 \cos \left(3\left(\theta_{1}+\theta_{2}\right)\right)+5 \cos \left(\theta_{1}-\theta_{2}\right)\right. \\
& \left.-\cos \left(5 \theta_{1}+\theta_{2}\right)+2 \cos \left(\theta_{1}+5 \theta_{2}\right)\right)
\end{aligned}
$$

and for $(X, Y, Z)=\left(e_{3}, e_{1}, e_{2}\right)$ we obtain

$$
\begin{aligned}
e_{3}\left(\alpha_{2}\right)=\frac{1}{4} & \alpha_{2} \alpha_{3} \csc \left(\theta_{1}+2 \theta_{2}\right)\left(-\cos \left(\theta_{1}-4 \theta_{2}\right)-7 \cos \left(3 \theta_{1}\right)+5 \cos \left(\theta_{1}+2 \theta_{2}\right)\right. \\
& \left.+\cos \left(3\left(\theta_{1}+2 \theta_{2}\right)\right)+2 \cos \left(5 \theta_{1}+4 \theta_{2}\right)\right) \\
e_{1}\left(\alpha_{2}\right)=- & \frac{1}{4} \alpha_{1} \alpha_{2} \csc \left(\theta_{1}-\theta_{2}\right)\left(-\cos \left(\theta_{1}+5 \theta_{2}\right)-7 \cos \left(3\left(\theta_{1}+\theta_{2}\right)\right)+5 \cos \left(\theta_{1}-\theta_{2}\right)\right. \\
& \left.+\cos \left(3\left(\theta_{1}-\theta_{2}\right)\right)+2 \cos \left(5 \theta_{1}+\theta_{2}\right)\right)
\end{aligned}
$$


Using these expressions for the derivatives of $\alpha_{1}, \alpha_{2}$ and $\alpha_{3}$, the $J e_{1}$-component of the Codazzi equation for $(X, Y, Z)=\left(e_{2}, e_{1}, e_{1}\right)$ yields $\alpha_{1} \alpha_{2}=0$, the $J e_{2}$-component of the Codazzi equation for $(X, Y, Z)=\left(e_{3}, e_{2}, e_{2}\right)$ yields $\alpha_{2} \alpha_{3}=0$ and the $J e_{3}$-component of the Codazzi equation for $(X, Y, Z)=\left(e_{1}, e_{3}, e_{3}\right)$ yields $\alpha_{1} \alpha_{3}=0$. This implies that at least two of the functions $\left\{\alpha_{1}, \alpha_{2}, \alpha_{3}\right\}$ vanish identically. Because of the symmetry of the problem, we may assume without loss of generality that $\alpha_{1}=\alpha_{2}=0$.

The $J e_{2}$-component of the Codazzi equation for $(X, Y, Z)=\left(e_{1}, e_{2}, e_{1}\right)$ then gives

$$
\alpha_{3}^{2}=2 \cos \left(\theta_{1}-\theta_{2}\right) \csc \left(3 \theta_{1}\right) \csc \left(3 \theta_{2}\right),
$$

whereas the $J e_{2}$-component of the Codazzi equation for $(X, Y, Z)=\left(e_{3}, e_{1}, e_{3}\right)$ gives

$$
\begin{aligned}
e_{3}\left(\alpha_{3}\right)= & \frac{1}{16} \csc \left(3 \theta_{1}\right) \csc \left(2 \theta_{1}+\theta_{2}\right) \csc \left(\theta_{1}+2 \theta_{2}\right)\left[\alpha_{3}^{2} \cos \left(2 \theta_{1}-5 \theta_{2}\right)+4 \alpha_{3}^{2} \cos \left(6 \theta_{1}-3 \theta_{2}\right)\right. \\
& -32 \cos \left(2 \theta_{1}+\theta_{2}\right) \sin \left(2 \theta_{1}+\theta_{2}\right)^{2}+\alpha_{3}^{2}\left[\cos \left(8 \theta_{1}+\theta_{2}\right)-16 \cos \left(4 \theta_{1}-\theta_{2}\right)\right. \\
& +15 \cos \left(2 \theta_{1}+\theta_{2}\right)+4 \cos \left(3\left(2 \theta_{1}+\theta_{2}\right)\right)-\cos \left(5\left(2 \theta_{1}+\theta_{2}\right)\right)-8 \cos \left(3 \theta_{2}\right) \\
& \left.\left.+2 \sin \left(3 \theta_{1}\right) \sin \left(5 \theta_{1}+7 \theta_{2}\right)\right]\right] .
\end{aligned}
$$

Substituting (27) and (28) in the Codazzi equation for $(X, Y, Z)=\left(e_{3}, e_{2}, e_{3}\right)$ gives

$$
-5 \cos \left(\theta_{1}-\theta_{2}\right)+2 \cos \left(3\left(\theta_{1}-\theta_{2}\right)\right)+\left(1+2 \cos \left(2\left(\theta_{1}-\theta_{2}\right)\right)\right) \cos \left(3 \theta_{1}+3 \theta_{2}\right)=0,
$$

while substituting them into the derivative of the Gauss equation for $(X, Y, Z, W)=\left(e_{1}, e_{2}, e_{2}, e_{1}\right)$ in the direction of $e_{3}$ yields

$$
1+2 \cos \left(2\left(\theta_{1}-\theta_{2}\right)\right)=0 .
$$

Combining these last two equations, we obtain that all angle functions are constant, again contradicting the result in [14].

\subsection{Classification in dimension $n \geq 4$.}

Proposition 6.7. For $n \geq 4$, let $f: M^{n} \rightarrow Q^{* n}$ be a minimal Lagrangian immersion such that the induced metric on $M^{n}$ has constant sectional curvature. Then $f$ is the Gauss map of a part of a standard embedding of $H^{n} \rightarrow H_{1}^{n+1}(-1)$.

Proof. Here we combine the proofs of Proposition 5.6 and Proposition 5.7 of [7], where we need to change the sectional curvature accordingly since the Gauss equation (7) for $Q^{* n}$ is similar to but different from the one for $Q^{n}$.

Combining Propositions 6.5, 6.6 and 6.7 finishes the proof of Theorem 6.1.

\section{REFERENCES}

1. M. Do Carmo and M. Dajczer, Rotation hypersurfaces in spaces of constant curvature, Trans. Amer. Math. Soc. 277 (1983), 685-709. MR 0694383

2. D. Gao, J. Van der Veken, A. Wijffels, and B. Xu, Lagrangian surfaces in $\mathbb{H}^{2} \times \mathbb{H}^{2}$, preprint.

3. H. Iriyeh, H. Ma, R. Miyaoka, and Y. Ohnita, Hamiltonian non-displaceability of Gauss images of isoparametric hypersurfaces, Bull. Lond. Math. Soc. 48 (2016), 802-812. MR 3556363

4. G. R. Jensen, Homogeneous Einstein spaces of dimension four, J. Differential Geom. 3 (1969), 309-349. MR 0261487

5. G. J. Kim and Y. J. Suh, Real hypersurfaces in the complex hyperbolic quadric with parallel Ricci tensor, Results Math. 74 (2019), Art. 33, 30 pp. MR 3902463

6. S. Klein and Y. J. Suh, Contact real hypersurfaces in the complex hyperbolic quadric, Ann. Mat. Pura Appl. (4) 198 (2019), 1481-1494. MR 3987223 
7. Haizhong Li, Hui Ma, Joeri Van der Veken, Luc Vrancken, and Xianfeng Wang, Minimal Lagrangian submanifolds of the complex hyperquadric, Sci. China Math. 63 (2020), no. 8, 1441-1462. MR 4125728

8. H. Ma and Y. Ohnita, Hamiltonian stability of the Gauss images of homogeneous isoparametric hypersurfaces. I, J. Differential Geom. 97 (2014), 275-348. MR 3263508

9. - Hamiltonian stability of the Gauss images of homogeneous isoparametric hypersurfaces II, Tohoku Math. J. (2) 67 (2015), 195-246. MR 3365370

10. S. Montiel and A. Romero, Complex Einstein hypersurfaces of indefinite complex space forms, Math. Proc. Cambridge Philos. Soc. 94 (1983), 495-508. MR 720800

11. M. Moruz and L. Vrancken, Warped product hypersurfaces in pseudo-Riemannian real space forms, Geometry of Submanifolds, Contemp. Math., vol. 756, Amer. Math. Soc., 2020, pp. 173-186.

12. B. Palmer, Hamiltonian minimality and Hamiltonian stability of Gauss maps, Differential Geom. Appl. 7 (1997), 51-58. MR 1441918

13. H. Reckziegel, Horizontal lifts of isometric immersions into the bundle space of a pseudo-Riemannian submersion, Global differential geometry and global analysis 1984 (Berlin, 1984), Lecture Notes in Math., vol. 1156, Springer, Berlin, 1985, pp. 264-279. MR 824074

14. V. Sanmartín-López, Spacelike isoparametric hypersurfaces, Differential Geom. Appl. 54 (2017), 53-58. MR 3693912

15. B. Smyth, Differential geometry of complex hypersurfaces, Ann. of Math. (2) 85 (1967), 246-266. MR 0206881

16. Y. J. Suh, Real hypersurfaces in the complex hyperbolic quadrics with isometric Reeb flow, Commun. Contemp. Math. 20 (2018), 1750031, 20 pp. MR 3730753

17. J. Van der Veken and A. Wijffels, Lagrangian submanifolds of the complex quadric as Gauss maps of hypersurfaces of spheres, Geometry of Submanifolds, Contemp. Math., vol. 756, Amer. Math. Soc., 2020, pp. 241-246.

Ku Leuven, Department of Mathematics, Celestijnenlaan 200B - Box 2400, 3001 Leuven, Belgium Email address: joeri.vanderveken@kuleuven.be

Email address: anne.wijffels@kuleuven.be 